\title{
Unsteady RANS and detached-eddy simulations of flow around a circular cylinder in ground effect
}

\author{
T. Nishino, G.T. Roberts*, X. Zhang \\ School of Engineering Sciences, University of Southampton, Southampton SO17 1BJ, UK \\ Received 28 September 2006; accepted 23 June 2007 \\ Available online 20 September 2007
}

\begin{abstract}
Unsteady Reynolds-averaged Navier-Stokes (URANS) simulations and detached-eddy simulations (DES) were performed of flow around a circular cylinder placed near and parallel to a moving ground, on which substantially no boundary layer developed to interfere with the cylinder. The results were compared with experiments previously reported by the authors to examine how accurately the URANS and DES can predict the cessation of von Kármán-type vortex shedding and the attendant critical drag reduction of the cylinder in ground effect. The DES, which were performed in a three-dimensional domain with spanwise periodicity imposed, correctly captured the cessation of the vortex shedding, whereas both two- and three-dimensional URANS also predicted it but at a much smaller gapto-diameter ratio compared with the experiments. The wake structures of the cylinder predicted by the DES were in good agreement with the experiments in both large- and small-gap regimes, and also in the intermediate-gap regime, where the DES captured the intermittence of the vortex shedding in the near-wake region. Based on the results obtained, further discussions are also given to the reason why the von Kármán-type vortices in the URANS solutions incorrectly 'survived' until the cylinder came much closer to the ground.
\end{abstract}

(C) 2007 Elsevier Ltd. All rights reserved.

Keywords: Unsteady RANS (URANS); Detached-eddy simulation (DES); Circular cylinder; Ground effect; Vortex shedding; Wake instability

\section{Introduction}

Large-scale, von Kármán-type (i.e., asymmetric, alternating) vortex shedding from cylindrical bluff bodies is of fundamental interest as well as of great importance in fluid mechanics. It is of crucial importance in many engineering applications to fully understand the characteristics of, and to accurately predict the behaviour of, this type of vortex shedding since its variation may significantly alter various fluid-mechanical properties of practical interest, such as flow-induced forces, vibrations and noise, and the efficiencies of heat and mass transfer. There are many cases in which a significant change can be seen in the behaviour of this type of vortex shedding, and one of the simplest - the mechanisms of which, however, are still unclear - is that observed when a circular cylinder is located near a plane boundary or ground; the focus of the present study is on this flow configuration.

\footnotetext{
*Corresponding author. Tel.: + 442380592325 ; fax: + 442380593058 .

E-mail address: gtr@soton.ac.uk (G.T. Roberts).
} 


\begin{tabular}{|c|c|c|c|}
\hline \multicolumn{2}{|c|}{ Nomenclature } & St & Strouhal number, $f d / U_{\infty}$ \\
\hline$B$ & wake width & $U, V, W$ & Cartesian components of velocity \\
\hline$C_{D}$ & drag coefficient & $U_{\infty}$ & free-stream velocity \\
\hline$C_{L}$ & lift coefficient & $x, y, z$ & Cartesian coordinates \\
\hline$C_{p}$ & pressure coefficient & $y^{+}$ & scaled wall distance \\
\hline CFL & Courant-Friedrichs-Lewy number & $\Delta t$ & time step \\
\hline$d$ & cylinder diameter & $\Delta x, \Delta y, \Delta z$ & size of control volume \\
\hline$f$ & frequency & & angle \\
\hline $\begin{array}{l}H \\
h\end{array}$ & $\begin{array}{l}\text { gap between wake centreline and ground } \\
\text { gap between cylinder and ground }\end{array}$ & $\theta_{\text {sep }}^{+}, \theta_{\text {sep }}^{-}$ & $\begin{array}{l}\text { separation angles }(+: \text { upper side, }- \text { : } \\
\text { bottom side) }\end{array}$ \\
\hline$L_{r}$ & recirculation length & $v$ & kinematic viscosity \\
\hline$n$ & nearest wall distance & $\omega_{z}$ & nondimensional spanwise vorticity, \\
\hline$\tilde{n}$ & length scale in DES & & $(\partial V / \partial x-\partial U / \partial y) d / U_{\infty}$ \\
\hline $\operatorname{Re}$ & Reynolds number, $U_{\infty} d / v$ & & \\
\hline
\end{tabular}

The flow around a circular cylinder placed near and parallel to a ground has been experimentally investigated by Bearman and Zdravkovich (1978), Zdravkovich (1985), Lei et al. (1999), and the present authors (Nishino et al., 2007), among others [see Nishino et al. (2007) for a more extensive review]. The characteristics of the flow and force acting on the cylinder in this case are governed not only by the Reynolds number Re but also by the gap ratio, i.e., the ratio of the distance between the cylinder and the ground, $h$, to the cylinder diameter $d$ (Bearman and Zdravkovich, 1978). However, the mechanisms of the flow and force variations caused by different $h / d$, or 'ground effect', are in general rather complicated since they can be significantly affected by the state of the boundary layer formed on the ground (Zdravkovich, 1985; Lei et al., 1999). Hence the present authors recently carried out a series of experiments on a circular cylinder placed near a moving ground running at the same speed as the free-stream $\left(\mathrm{Re}=0.4\right.$ and $1.0 \times 10^{5}$ based on the cylinder diameter d) to avoid the confusing effects of the boundary layer and thereby to elucidate the essence of the ground effect (Nishino et al., 2007). The cylinder was equipped with a pair of end-plates to ensure quasi-two-dimensionality of the flow. As a result, the characteristics of the flow were classified into three fundamental regimes: large-gap $(h / d>0.5)$, intermediate-gap $(0.35<h / d<0.5)$, and small-gap $(h / d<0.35)$ regimes. In the large-gap regime, large-scale, von Kármán-type vortices were generated just behind the cylinder, whereas in the small-gap regime, the vortex shedding ceased and instead a dead-fluid zone was created, bounded by two nearly parallel shear layers each producing only small-scale vortices, as shown in Fig. 1. Also of particular interest in the study was that the drag coefficient of the cylinder rapidly decreased as $h / d$ decreased from 0.5 to 0.35 but became constant for $h / d<0.35$, suggesting that the rapid drag reduction of the cylinder in ground effect is primarily related to the cessation of the von Kármán-type vortex shedding in the near-wake region.

(a)

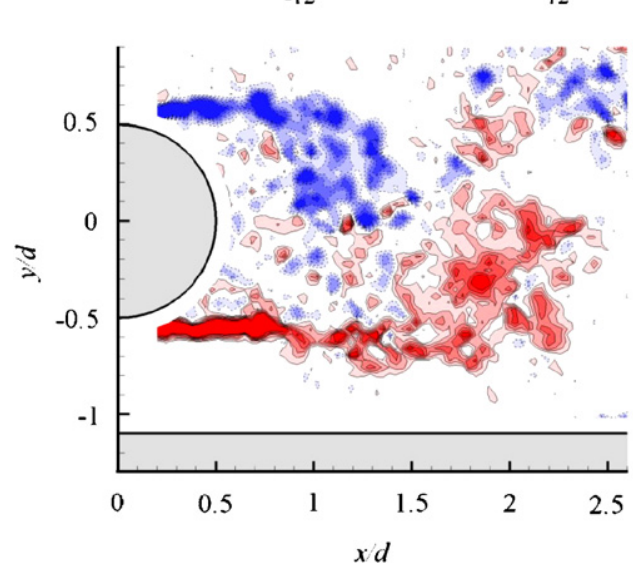

(b)

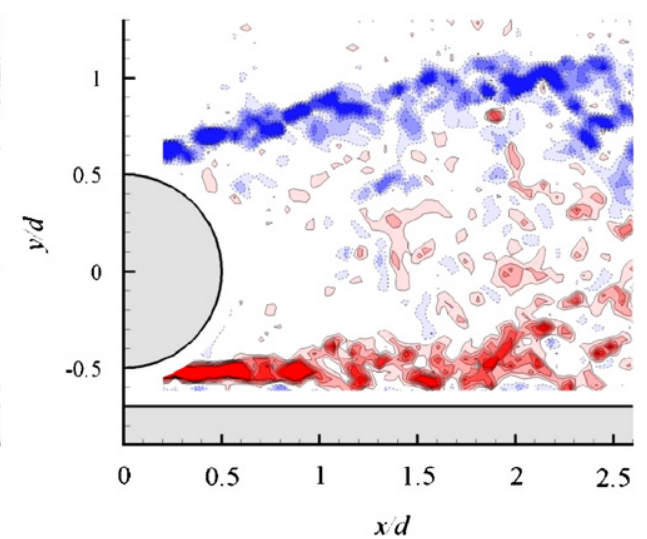

Fig. 1. Typical instantaneous wake structures (spanwise vorticity fields) behind a circular cylinder in ground effect, measured by PIV, $\mathrm{Re}=4.0 \times 10^{4}$ [reproduced from Nishino et al. (2007)]. (a) $h / d=0.6$; (b) $h / d=0.2$. 
The numerical investigation reported in this paper is a subsequent study of the experiments described above. The flow around a circular cylinder at Reynolds numbers greater than $10^{3}-10^{4}$, however, is still a challenging subject in today's computational fluid dynamics (CFD) even if the cylinder is outside the ground effect. Steady and even unsteady Reynolds-averaged Navier-Stokes (SRANS and URANS, respectively) simulations cannot reproduce with sufficient accuracy the wake structures of wide-ranging spatial and time scales, whereas direct numerical simulations (DNS) of this type of flow are not currently feasible due to the huge computational costs required (Spalart, 2000). A further difficulty for simulating the flow around a circular cylinder arises from the fact that the flow separation points are not fixed or determined by the geometry (Shur et al., 1996), in comparison with, for example, a square cylinder, for which the separation points are fixed at the two front corners and the flow can be reasonably predicted, at least in the mean sense, by URANS [with a special care to avoid the excessive production of turbulence in the front stagnation region, e.g., Kato and Launder (1993), Bosch and Rodi (1998); see also Section 3.3]. Some promising results of large-eddy simulations (LES) have been reported by Breuer $(1998,2000)$ on a circular cylinder in a uniform cross-flow $\left(\operatorname{Re}=3.9 \times 10^{3}\right.$ and $1.4 \times 10^{5}$, respectively), but an essential problem in the use of LES is again the computational cost required to sufficiently resolve the near-wall region, especially in high-Re cases. A possible alternative to LES is the detached-eddy simulation (DES), which is one of the hybrid methods that combine URANS and LES to obtain realistic solutions of practical high-Re flows at acceptable computational costs [Shur et al. (1999); see also Breuer et al. (2003) and Squires (2004) for recent progress on DES]. Travin et al. (2000) performed the DES of flow around a circular cylinder in a uniform cross-flow in the subcritical (laminar-separation) and postcritical (turbulent-separation) flow regimes. The results were in good agreement with experiment and also with LES, especially at a subcritical Reynolds number of $5.0 \times 10^{4}$.

The objective of the present study, where the flow around a circular cylinder placed near a moving ground is numerically simulated, is twofold. The first goal is to examine whether, and how accurately, URANS and DES can reproduce the cessation of the von Kármán-type vortex shedding and consequently the rapid or critical drag reduction of the cylinder in ground effect. This flow configuration might be considered, from the viewpoint of CFD researchers, as one of the simplest (but still challenging) test cases that contain the problem of large-scale vortex shedding and its cessation or suppression, and hence the results of the present validation may serve as a primary criterion for the applicability of URANS and DES to such vortical flows often encountered in engineering applications. The second objective, on the other hand, is to investigate the predicted flow fields in more detail, and thus to provide further insight into the physical mechanisms of the ground effect as well as into the reason why the DES and URANS can/cannot correctly capture the cessation of the vortex shedding. The DES in the present study, which is based on the Spalart-Allmaras one-equation model [S-A model; Spalart and Allmaras (1992)], is performed in a three-dimensional domain with periodicity imposed in the spanwise direction, basically following the DES by Travin et al. (2000). Meanwhile, the URANS simulations are carried out in both two- and three-dimensional domains, where the S-A model and also a classical, low-Reynolds-number $k-\varepsilon$ model of Launder and Sharma [LS $k-\varepsilon$ model; Launder and Sharma (1974)] are employed for the purpose of comparison. The results reported in this paper are restricted to a subcritical flow of $\mathrm{Re}=4.0 \times 10^{4}$ to focus on the issues relevant to the ground effect rather than to the Reynolds number effect.

\section{Computational details}

\subsection{Overview}

Fig. 2 summarises the computational domain and boundary conditions employed in this study; the ground effect was simulated by changing the gap ratio $h / d$ from 1.0 to 0.1 . The computations were conducted using a commercial CFD package, FLUENT6 (Fluent Inc., 2005), in which a finite volume method was used to discretise the governing/model equations for incompressible turbulent flows to be solved. The equations were spatially discretised with second-order accuracy on multi-block structured grids (Fig. 3), temporally discretised using a second-order fully implicit scheme, and then iteratively solved. Further details of the computation are described below.

\subsection{URANS simulation}

The concept of URANS is based on the triple decomposition of time-dependent variables (Hussain and Reynolds, 1970), where each instantaneous variable, such as velocity and pressure, is decomposed into long-time-averaged, periodic, and turbulent (or stochastic/random) components. The sum of the first two is often referred to as a coherent (or ensemble-averaged) component, for which the Reynolds-averaged Navier-Stokes (RANS) equations are derived 


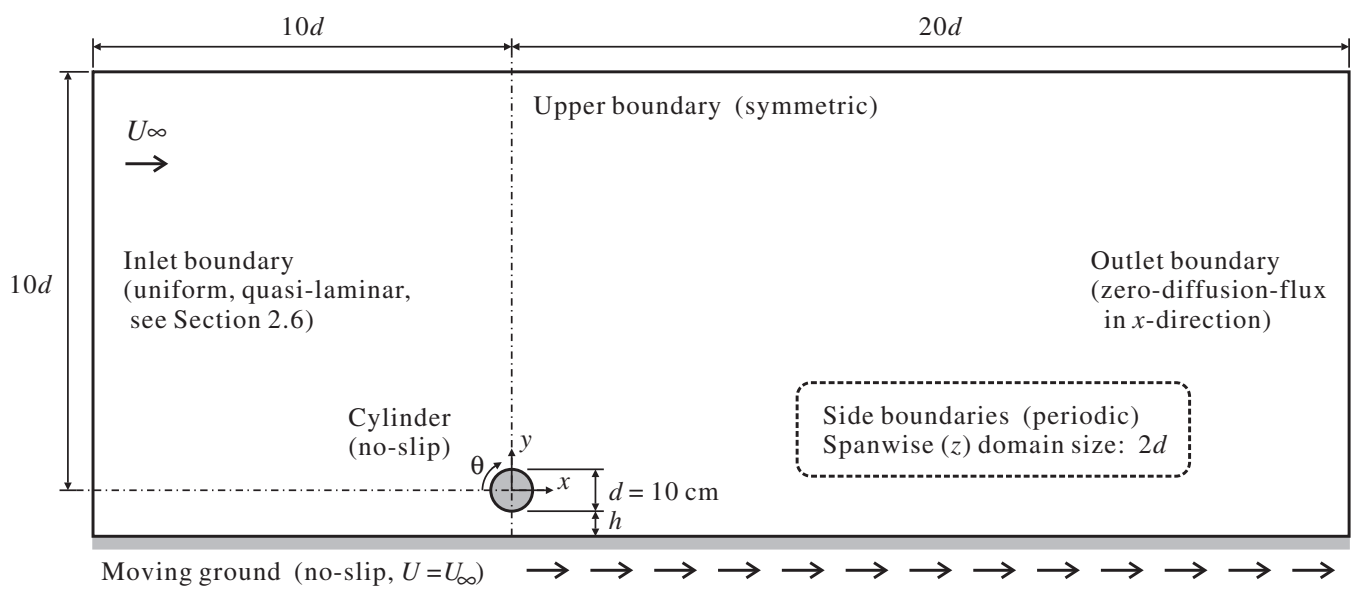

Fig. 2. Computational domain and boundary conditions.

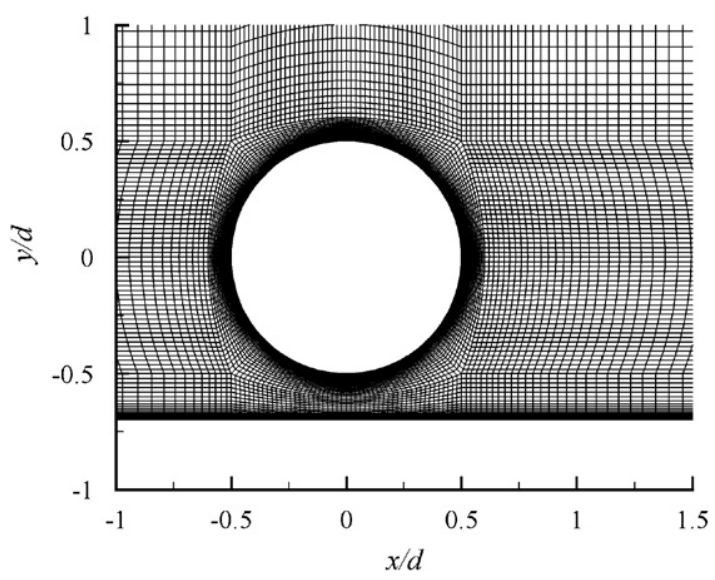

Fig. 3. Example of computational grids $(h / d=0.2)$.

and solved by using some turbulence closure models. It should be noted, however, that most closure models of today, including the S-A and LS $k-\varepsilon$ models used in this study, are basically adjusted to reproduce the spreading rate of mixing layers in steady RANS simulations, i.e., the Reynolds stresses predicted by the models implicitly contain contributions not only from the turbulent or random motions but also from the coherent motions of the flow (Spalart, 2000), and therefore, in general, the models are 'overly dissipative' (Menter et al., 2003) when used in URANS simulations. It should also be noted that, in most real flows, including the cylinder wakes studied here, the turbulent and coherent motions cannot be explicitly distinguished as the scales of the two motions do not differ enough in nature.

\subsection{Detached-eddy simulation}

The main feature of the DES performed in this study is that a single turbulence model (a slightly modified version of the S-A model) serves as a statistical model (i.e., URANS mode) in near-wall regions, and also serves as a subgrid-scale model (i.e., LES mode) in far-wall regions. Specifically, the nearest-wall-distance $n$ that governs the eddy viscosity in the original S-A model is replaced in the DES by a new length scale $\tilde{n}$ defined as

$$
\tilde{n}=\min \left[n, 0.65 \Delta_{\max }\right], \quad \Delta_{\max }=\max [\Delta x, \Delta y, \Delta z],
$$

where $\Delta x, \Delta y$ and $\Delta z$ denote the size of a control volume (or the grid spacing) in each direction. This simple formulation of the DES, however, raises an issue concerning the physical interpretation of the 'grey area' $\left(n \approx 0.65 \Delta_{\max }\right)$, where the 
operation mode is switched between URANS and LES, and the justification of this switch solely relies on the disparity in the scales between the attached- and detached-eddies (Spalart, 2001). In practice, the switch between the URANS and LES modes can take place inside the boundary layers when using excessively fine computational grids, and this often causes a premature or grid-induced separation (Menter et al., 2003). It follows that the grid spacing in DES should be carefully decided so that the boundary layers are analysed in the URANS mode rather than in the LES mode.

\subsection{Computational grids}

Two- and three-dimensional multi-block structured grids were created in this study, the former of which were only for URANS and the latter of which were for both URANS and DES. An example of the two-dimensional grids is shown in Fig. 3, where the gap ratio $h / d=0.2$. This grid topology, which basically follows that used for a circular cylinder in free-stream by Menter et al. (2003), was employed because of its simplicity and applicability to the current flow configuration (i.e., with a nearby ground). Note that this grid topology is not 'optimal' in the sense that the angles between grid lines change on the block interfaces; this, however, did not appear to cause any problems or influence the results significantly in the current work. In order to investigate the ground effect, 14 different grids (seven for twodimensional and the other seven for three-dimensional cases, for $h / d=0.1,0.2,0.3,0.4,0.5,0.6$, and 1.0 , respectively) were created but keeping the same grid topology.

For the two-dimensional $(x-y)$ grids, 200 grid points were equidistantly located around the cylinder, whereas 180 points were non-equidistantly distributed on the ground. The distance from the cylinder and ground surfaces to the nearest grid points is $0.0002 d$, which ensures a sufficient spatial resolution of $y^{+} \leqslant 0.6$ in this study. The number of the grid points for the two-dimensional grids ranges from 27200 (for $h / d=0.1$ ) to 35300 (for $h / d=1.0$ ). The threedimensional grids were then created by simply extending the two-dimensional grids in the spanwise $(z)$ direction. The spanwise length of the domain was set at $2 d$ with periodic boundary conditions imposed on the ends, following the DES by Travin et al. (2000), and 40 grid points were equidistantly located in that direction, i.e., the total number of the grid points ranges from about 1.1 to 1.4 million (depending on $h / d$ ), and the spanwise grid spacing $\Delta z=0.05 d$. It should be noted that this spanwise grid spacing was selected considering the thickness of the boundary layer on the cylinder surface (about $0.03 d$ at the maximum) since $\Delta z$ is the largest grid spacing (i.e., larger than $\Delta x$ and $\Delta y$ ) around the cylinder and hence in the DES directly affects the switching between the URANS and LES modes in that region [cf. Eq. (1)]. Specifically, for the region around the cylinder, a thin URANS layer of $0.0325 d$ thickness was formed, in which 30 grid points were allocated in the direction normal to the cylinder surface.

In addition to these 14 grids for the main part of the study, another five grids of different spatial resolutions (in the $x-y$ plane and also in the $z$ direction) were also created to examine the grid dependency of the simulations. Although a problem of explicit grid dependency in DES has been pointed out by Menter et al. (2003) and other researchers, a certain degree of grid convergence was achieved in this study due to the rather simple geometry of the flow simulated; details will be described later in the Appendix.

\subsection{Numerical methods and procedures}

The governing/model equations derived from the URANS and DES formulations were spatially discretised on the structured grids described above. Basically the convection terms were discretised using a second-order upwind scheme, whereas the diffusion terms were discretised using a second-order central difference scheme. An exception to this, however, was the convection term in the momentum equations in the DES, for which the central difference scheme was used rather than the upwind scheme, in order to reduce the influence of numerical diffusion [see, e.g., Ferziger and Peric (2002)]. The equations were further discretised in time by using a second-order (or three-time-level) fully implicit scheme, and then iteratively solved using the 'segregated' solver in FLUENT6 (Fluent Inc., 2005), in which the SIMPLE algorithm (Patankar, 1980) was used to derive the pressure-correction equations.

For most computations in this study, 20-30 iterations were found to be enough for the solution at each time instant to be converged. The dimensionless time step $\Delta t U_{\infty} / d$ was set at 0.021 , which ensured sufficiently small CFL numbers of less than 2 for most part of the computational domain. A smaller $\Delta t U_{\infty} / d$ of 0.0042 was preliminarily tested for comparison, and the differences between the two cases were found to be very small, as shown in the Appendix. The iteration process was first continued until the flow field had sufficiently developed, i.e., the influence of initial conditions had become negligible, and then was further continued to obtain the time-averaged data of the flow field. At least 100 dimensionless time units $\left(t U_{\infty} / d\right)$, which corresponded to about 20-30 vortex-shedding cycles, were taken at this stage so as to obtain reliable statistical information. 


\subsection{Boundary conditions}

At the inlet boundary of the domain (see Fig. 2), a steady uniform flow $\left(U_{\infty}=6 \mathrm{~m} / \mathrm{s}\right)$ of low turbulence level (corresponding to a turbulence intensity of $0.3 \%$ and a turbulent viscosity ratio of unity, with the former condition applicable only to the LS $k-\varepsilon$ URANS) was given so as to simulate the subcritical flow $\left(\mathrm{Re}=4.0 \times 10^{4}\right)$ around the cylinder. For the DES and S-A URANS simulations, however, the so-called 'trip-less approach' (TLA) was additionally used, following Travin et al. (2000), in order to obtain more 'plausible' results for the subcritical flow of this Re range [i.e., the transition to turbulence taking place just after the flow separation from the cylinder, see, e.g., Zdravkovich (1997)]. Specifically, the turbulent viscosity ratio at the inlet was reduced from unity to $10^{-9}$ after the flow field had sufficiently developed under the original boundary conditions. This modification provides a 'self-sustaining' eddy-viscosity field behind the flow separation from the cylinder, i.e., the (almost) zero eddy viscosity values from the inlet propagates to the region before the separation but the nonzero values behind the separation are sustained as they diffuse into the separated shear layers and cause a rapid production of themselves, and thus the sudden increase in the eddy viscosity after the flow separation can be imitated without giving any information on the transition point a priori. After the change of the inlet turbulent viscosity ratio, the computation was further continued until the flow field had developed again, and thereafter the time-averaged data of the flow were collected.

At the outlet boundary, a condition of zero-diffusion-flux in the streamwise direction (which assumes no velocity gradient in that direction and is referred to as the 'outflow' boundary condition in FLUENT6) was applied. It was justified because the distance from the cylinder to the outlet boundary was sufficiently long for the outlet boundary condition to have negligible influence on the near-wake region of the cylinder flow. As concerns the upper boundary of the domain (cf. Fig. 2), a symmetry (or slip) condition was imposed also because the distance from the cylinder to the boundary was long enough. For the side boundaries, periodic conditions were imposed as mentioned before. As for the cylinder and ground surfaces, no-slip conditions $\left(U=V=W=0\right.$ for the cylinder, whereas $U=U_{\infty}$ and $V=W=0$ for the moving ground) were employed.

\section{Results and discussion}

Computational conditions are summarised in Table 1. Four types of simulations were performed in this study, hereafter simply referred to as '2-D $k-\varepsilon$ ', '2-D S-A', '3-D S-A', and 'DES'. Note that the 2-D $k-\varepsilon$ and 2-D S-A were carried out on the same two-dimensional grids, whereas the 3-D S-A and DES were performed on the same threedimensional grids, for $h / d=0.1,0.2,0.3,0.4,0.5,0.6$, and 1.0 , respectively.

\subsection{Drag reduction and the cessation of von Kármán-type vortex shedding}

Fig. 4 compares the time-averaged drag coefficient of the cylinder in ground effect. The results of the experiments conducted at the same Reynolds number (Nishino et al., 2007) are also shown in the figure for comparison. An important feature of the ground effect to be focused on here is the critical change in $C_{D}$ due to the cessation of the largescale von Kármán-type vortex shedding at $h / d<0.35$ (as illustrated in Fig. 1). As can be seen from the figure, both 2-D and 3-D URANS predicted a critical change in $C_{D}$ but at much smaller $h / d$ of $0.2-0.1$ (as they incorrectly predicted the large-scale vortex shedding at $h / d$ of down to 0.2 , as will be shown later). Meanwhile, the drag behaviour predicted by the DES agreed better with the experiments: the critical drag reduction was qualitatively correctly captured at $h / d$ of $0.4-0.3$, although the predicted values of $C_{D}$ were slightly higher than the experimental values at all $h / d$ investigated.

Table 1

Summary of computational conditions

\begin{tabular}{lllll}
\hline Case & Type & Model & TLA $^{\mathrm{a}}$ & $h / d$ \\
\hline 2-D $k-\varepsilon$ & 2-D, URANS & LS $k-\varepsilon$ & No & \\
2-D S-A & 2-D, URANS & S-A & Yes & $0.1,0.2,0.3,0.4,0.5,0.6,1.0$ \\
3-D S-A & 3-D, URANS & S-A & Yes & \\
DES & 3-D, DES & (Modified) S-A & Yes & \\
\hline
\end{tabular}

${ }^{\mathrm{a}}$ TLA: Trip-less approach (cf. Section 2.6). 


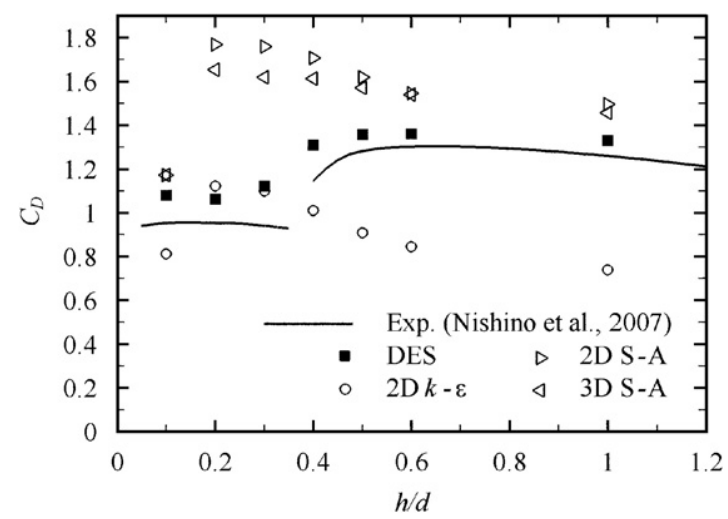

Fig. 4. Time-averaged drag coefficient versus gap ratio.

(a)

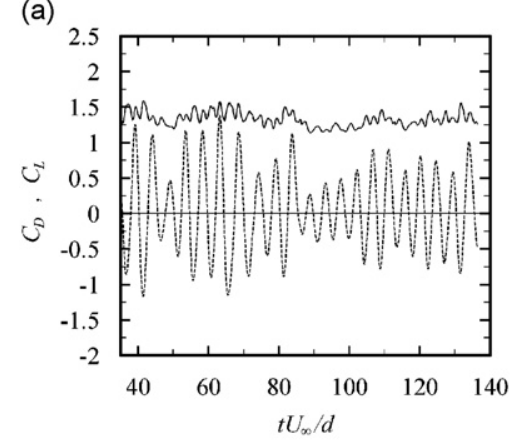

(d)

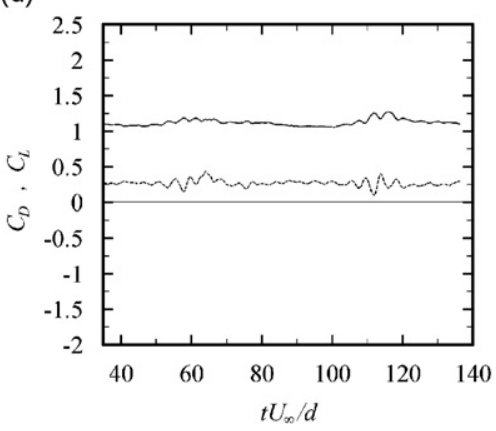

(b)

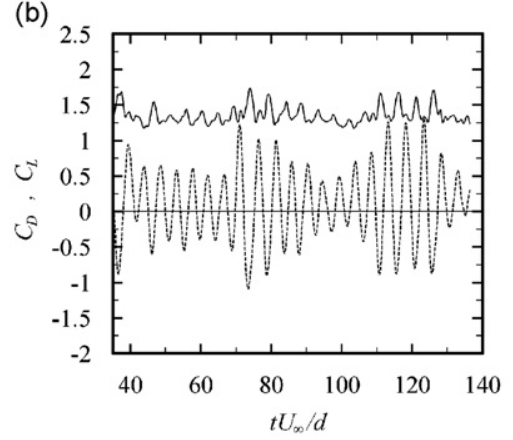

(e)

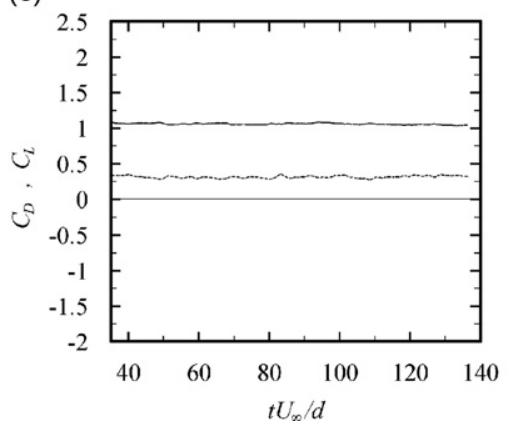

(c)

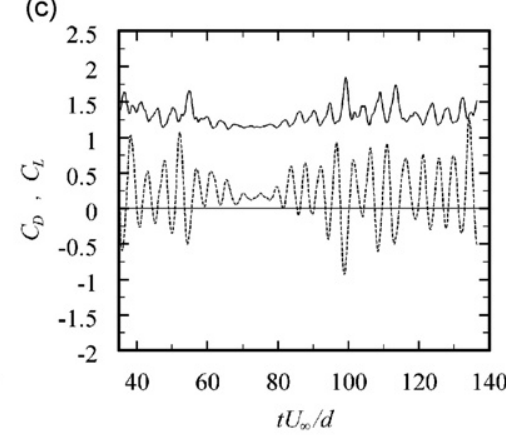

(f)

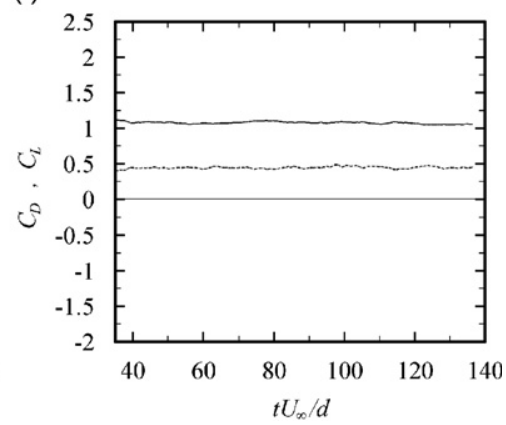

Fig. 5. Time variation of drag and lift coefficients (DES); solid and dashed lines indicate $C_{D}$ and $C_{L}$, respectively. (a) $h / d=1.0$; (b) $h / d=0.6$; (c) $h / d=0.4$; (d) $h / d=0.3$; (e) $h / d=0.2$; (f) $h / d=0.1$.

Fig. 5 shows the time variations of the drag and lift coefficients of the cylinder predicted by the DES for different gap ratios. Note that the solid and dashed lines indicate the variations of $C_{D}$ and $C_{L}$, respectively. It is obvious from the figures that the DES captured the cessation of the large-scale vortex shedding between two $h / d$ of 0.4 and 0.3 , which is consistent with the experiments. The Strouhal number St obtained was around $0.21-0.22$ for all $h / d$ of greater than 0.3 , which is comparable to the well known St value of about 0.2 for a circular cylinder outside the ground effect in this Re regime [see e.g., Zdravkovich (1997)]. Fig. 6 shows typical instantaneous wake structures of the cylinder predicted by the DES. A clear difference can be seen between the two cases: three-dimensional large-scale vortex shedding was predicted behind the cylinder at $h / d=0.6$, whereas two nearly parallel shear layers (but still having embedded threedimensional turbulent structures) were formed at $h / d=0.2$. The mid-span sections of these two instantaneous flow fields are shown in Fig. 7(a) and (b), respectively, where the contours of nondimensional spanwise vorticity are plotted. A comparison with the PIV results (Fig. 1) suggests that the DES successfully captured the main features of the wake structure of the cylinder in both the large- and small-gap regimes. 

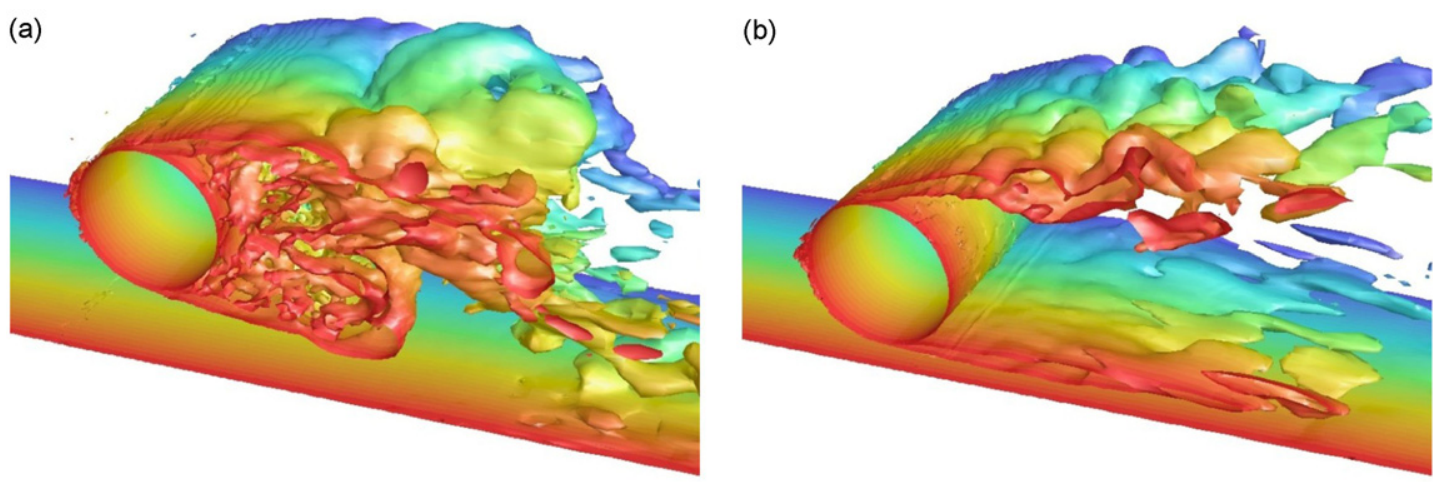

Fig. 6. Isosurfaces of the magnitude of instantaneous vorticity (DES). (a) $h / d=0.6$; (b) $h / d=0.2$.

(a)

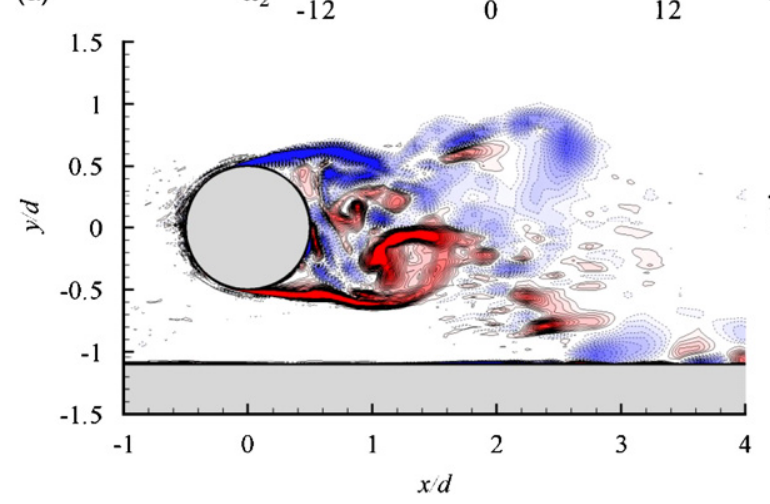

(b)

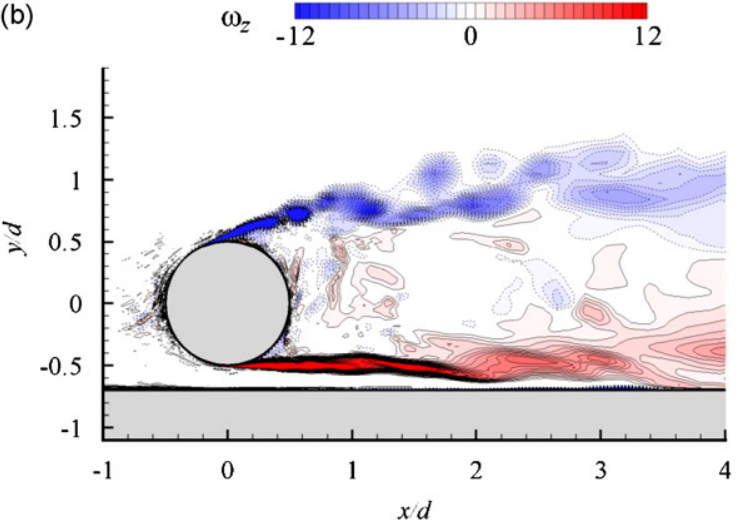

Fig. 7. Typical instantaneous spanwise vorticity fields at two different gap ratios (DES). (a) $h / d=0.6$; (b) $h / d=0.2$.

Of further interest in the time variation of the flow predicted by the DES is that the periodic shedding temporarily ceased at $h / d=0.4$ [Fig. 5(c), at $t U_{\infty} / d \approx 75$ ], and was only temporarily awakened at $h / d=0.3$ [Fig. 5(d), at $t U_{\infty} / d \approx$ 60 and 110]. This qualitatively agrees with the experimental observation that the von Kármán-type vortex shedding was intermittent in the intermediate gap regime (Nishino et al., 2007). Fig. 8 shows instantaneous spanwise vorticity fields behind the cylinder at $h / d=0.4$ at two different time instants. It can be seen that at $t U_{\infty} / d=75.6$ [Fig. 8(a)], around which time the fluctuations of the forces almost diminished to zero [cf. Fig. 5(c)], a small dead-fluid zone was temporarily generated behind the cylinder. At $t U_{\infty} / d=136.5$ [Fig. 8(b)], however, large-scale vortices were formed just behind the cylinder, similar to those observed at $h / d=0.6$ [cf. Fig. 7(a)].

Figs. 9 and 10 show the time variations of the force coefficients predicted by the 2-D and 3-D S-A simulations, respectively, at $h / d=1.0,0.4$, and 0.2 . The figures clearly show that, in contrast to the DES, both 2-D and 3-D S-A predicted a periodic vortex shedding even at $h / d=0.2$, where the shedding is supposed to cease according to the experiments (note that at $h / d=0.1$ the vortex shedding ceased and a steady solution was obtained in both 2-D and 3-D S-A and also in the 2-D $k-\varepsilon$ ). The Strouhal number obtained from the 2-D S-A was slightly higher than those from the 3-D S-A and DES (cf. Table 2 in the Appendix). Also of interest here is that the 2-D S-A (and also the 2-D $k-\varepsilon$ ) yielded fully periodic time variations at all $h / d$ of greater than 0.1 , whereas the $3-\mathrm{D}$ S-A showed some low-frequency modulations but only at $h / d \geqslant 0.4$ [cf. Fig. 10(a) and (b)]. Fig. 11 shows instantaneous wake structures of the cylinder predicted by the 3-D S-A at $h / d=0.6$ and 0.2. It can be seen that at $h / d=0.2$ the 3-D S-A yielded an almost twodimensional wake, whereas at $h / d=0.6$ it yielded a three-dimensional wake consisting of not only spanwise vortices but also streamwise 'rib' vortices, although these three-dimensional vortical structures are still much coarser than those predicted by the DES [cf. Fig. 6, see also Travin et al. (2000) for a circular cylinder in free-stream]. In this connection, Shur et al. (2005) recently reported, for a circular cylinder in free-stream, that this type of low-frequency modulation and moderate three-dimensionality in 3-D URANS may or may not be observed depending on the spanwise size of the 
(a)

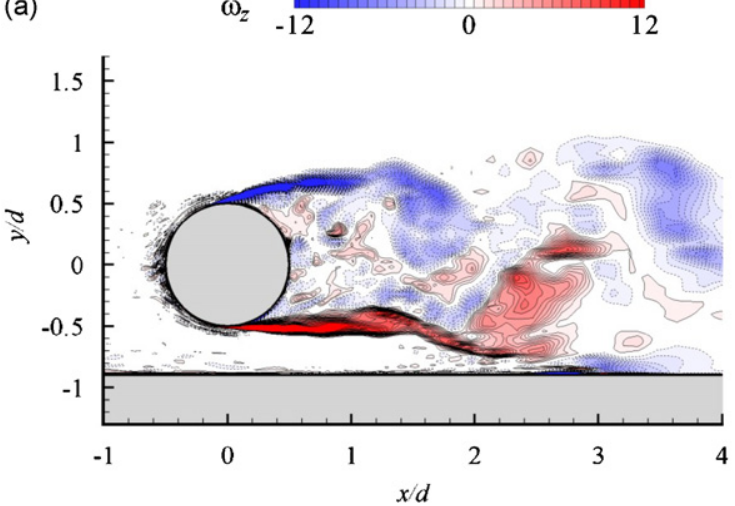

(b)
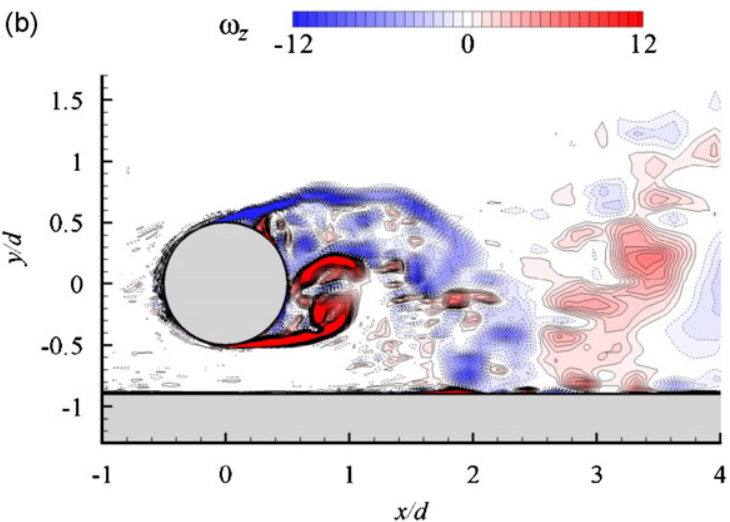

Fig. 8. Instantaneous spanwise vorticity fields at two different phases at $h / d=0.4$ : (a) $t U_{\infty} / d=75.6$ and (b) $t U_{\infty} / d=136.5$ (DES).
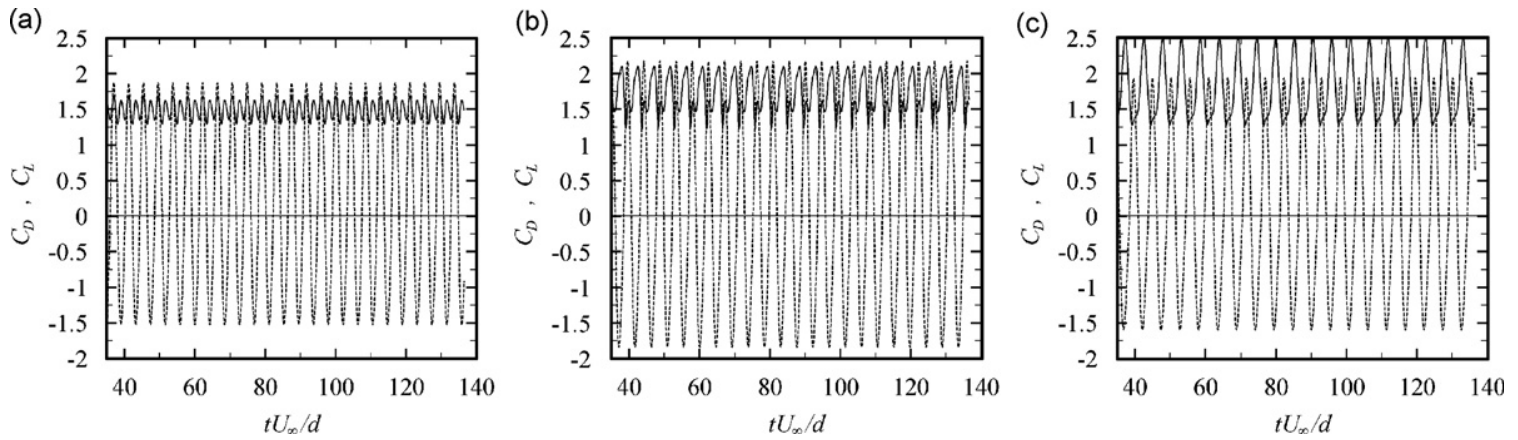

Fig. 9. Time variation of drag and lift coefficients (2-D S-A); solid and dashed lines indicate $C_{D}$ and $C_{L}$, respectively. (a) $h / d=1.0$; (b) $h / d=0.4$; (c) $h / d=0.2$.
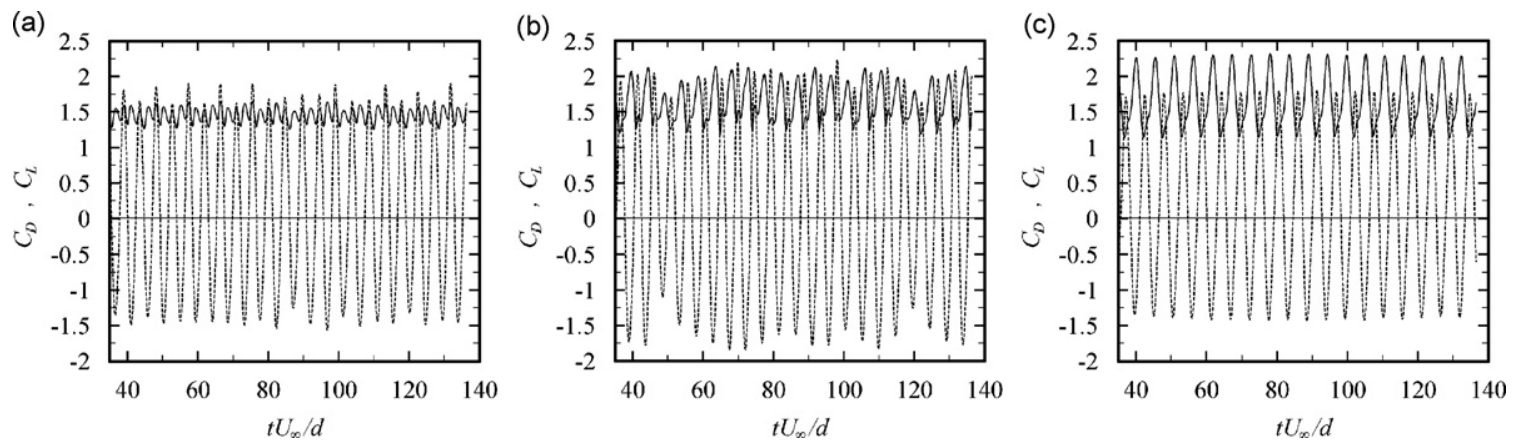

Fig. 10. Time variation of drag and lift coefficients (3-D S-A); solid and dashed lines indicate $C_{D}$ and $C_{L}$, respectively. (a) $h / d=1.0$; (b) $h / d=0.4$; (c) $h / d=0.2$.

domain and also on the turbulence model employed. The present results are of interest in showing that it may also depend on a geometric parameter of the flow simulated, even though the relevance of this to real physics is still unclear.

\subsection{Mean flow characteristics}

Fig. 12 compares the time-averaged separation angles on both upper (open) and bottom (gap) sides of the cylinder in ground effect. The separation angles estimated from oil flow visualisation tests [at a higher Reynolds number of 
$1.0 \times 10^{5}$ (Nishino et al., 2007)] are also shown here for the purpose of comparison (note that this Reynolds number in the oil flow tests is still within the subcritical regime and thus comparing the results should still be reasonable). Also note that $\left|\theta_{\text {sep }}\right|$ plotted in this figure indicates the magnitude of the angle from the front position $(x / d=-0.5, y / d=0)$ to the separation position. It can be seen that the DES agreed better with the experiments, whereas the URANS simulations predicted larger angles compared with the experiments (note that the angles predicted by the 2-D S-A, not presented here, were slightly larger compared with those by the 3-D S-A; cf. Table 2 in the Appendix).

Fig. 13 shows the mean pressure distributions around the cylinder predicted by the DES for different gap ratios. These results describe the mechanisms of the drag and lift behaviours in ground effect. That is, the rapid drag reduction at $h / d=0.4-0.3$ occurs primarily due to an increase in the base pressure, whilst the lift gradually increases as the gap decreases mainly because the high pressure region around the front stagnation point shifts to the bottom side of the cylinder. There are, unfortunately, no experimental data available to be compared with, although a good accuracy of DES on a pressure distribution around a circular cylinder in free-stream (i.e., outside the ground effect) has been reported by Travin et al. (2000) and also confirmed by the present authors (not presented here).

Fig. 14 show the time-averaged streamwise velocity contours obtained by the DES and 3-D S-A simulations. It can be seen that the DES predicted the recirculation region behind the cylinder to be significantly elongated as $h / d$ decreased from 0.4 to 0.3 and below, due to the cessation of the von Kármán-type vortex shedding. This agrees with the results of the PIV measurements performed at the same Reynolds number (Nishino et al., 2007), and is consistent with the increase in the base pressure described above. In contrast, the recirculation region predicted by the 3-D S-A remains small even at $h / d=0.2$, primarily because the 3 -D S-A failed to capture the cessation of the vortex shedding as mentioned before. Fig. 15 compares the length of the recirculation region, $L_{r}$, predicted behind the cylinder. The results from the PIV measurements are also plotted here for comparison. As is obvious from the figure, all URANS simulations in this study predicted smaller $L_{r}$ compared with those by the DES and the experiments, especially at smaller gap ratios.

Fig. 16 compares the mean streamwise velocity profiles behind the cylinder at $h / d=0.6$ and 0.2 . Note that the profiles at $x / d=0.5,1.0,1.5,2.0$, and 2.5 predicted by the DES and 3-D S-A are shown in each figure, and those obtained by the PIV measurements (but only at $x / d=1.0,1.5,2.0$, and 2.5 ) are also plotted for comparison. It can be seen that the DES successfully captured the mean wake profile of the cylinder at both larger and smaller gap ratios, i.e., the parabolic-like profiles because of the vortex shedding at $h / d=0.6$ and the top-hat-like profiles due to the cessation of the vortex shedding at $h / d=0.2$. In contrast, the 3-D S-A showed some discrepancies in the near-wake region at $h / d=0.6$, but totally failed to predict the correct profile at $h / d=0.2$, again primarily because the 3-D S-A failed to capture the cessation of the vortex shedding at this smaller gap ratio. This was also the case with the 2-D URANS simulations (not presented here).

\subsection{Further discussion on the cessation of von Kármán-type vortex shedding}

After comparing the accuracy of the URANS and DES in the preceding sections, the focus of the discussion is now back to the cessation of the von Kármán-type vortex shedding. As demonstrated in this study the capability of turbulence models/techniques to correctly predict the formation and cessation of large-scale vortex shedding is of crucial importance when they are applied to this type of bluff body flow. It is therefore worthwhile - not only from the physical but also from the computational point of view_to address the question: "Why did the von Kármán-type vortices incorrectly 'survive' in the URANS simulations until the cylinder came much closer to the ground?"

The first thing to be noted here is that the incorrect shedding observed in the URANS in this study contradicts the general view in CFD that vortex shedding should tend to be suppressed when the predicted flow is 'overly dissipative' (cf. Section 2.2) or the predicted eddy viscosity is excessively large. In fact, comparing the results between the 3-D S-A URANS and DES, the URANS yielded a much larger eddy viscosity than that by the DES in the region after the separation from the cylinder (note that in the region before the separation the eddy viscosity was kept almost zero in both URANS and DES due to the TLA, cf. Section 2.6). This shows that the accuracy of the prediction of the vortex shedding cannot be simply or directly linked with the difference in the level of eddy viscosity predicted.

A possible explanation for the 'delayed' cessation in the URANS, however, can be found in the mean wake profiles predicted by the URANS and DES at the smallest gap ratio of 0.1 , where not only the DES but also the URANS captured the cessation of the vortex shedding due to the strong influence of the nearby ground. Fig. 17 compares the mean streamwise velocity profiles at $x / d=1.0$ and 1.5 predicted by the 3-D S-A URANS and DES for $h / d=0.1$. It can be seen that the DES predicted a wider top-hat-like wake profile with a smaller backflow velocity in this region, whereas the 3-D S-A still yielded a narrower parabolic-like wake profile with a larger backflow velocity despite having captured the cessation of the vortex shedding. This difference in the mean velocity profiles in the near-wake region may be of crucial importance when considering the stability characteristics of the wake. 

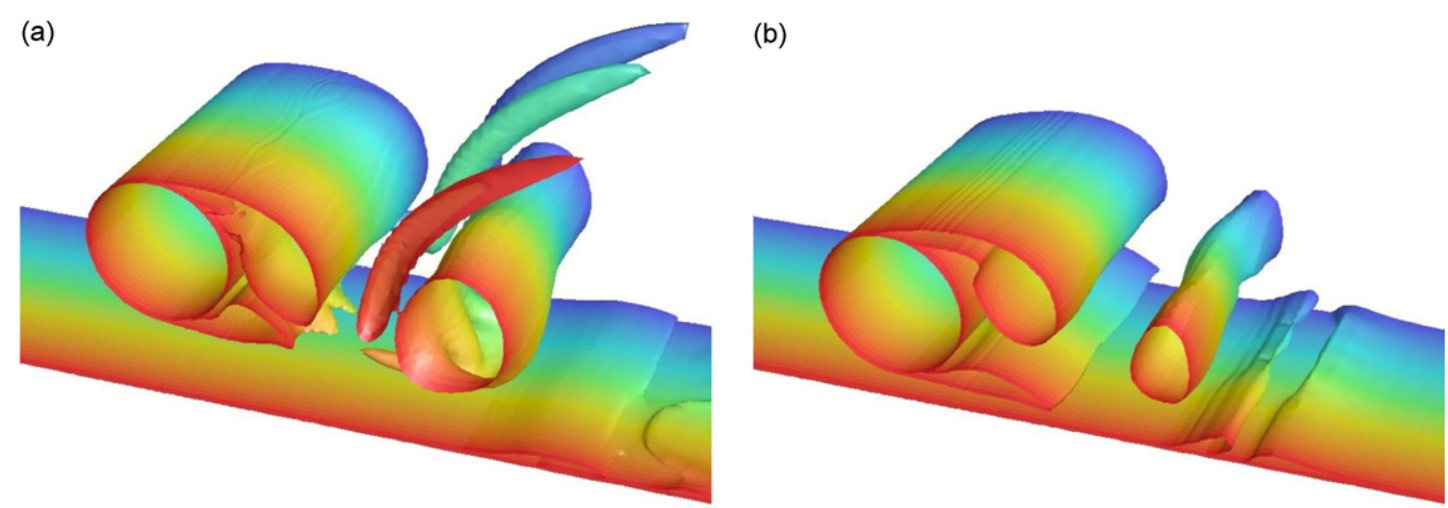

Fig. 11. Isosurfaces of the magnitude of instantaneous vorticity (3-D S-A). (a) $h / d=0.6$; (b) $h / d=0.2$.

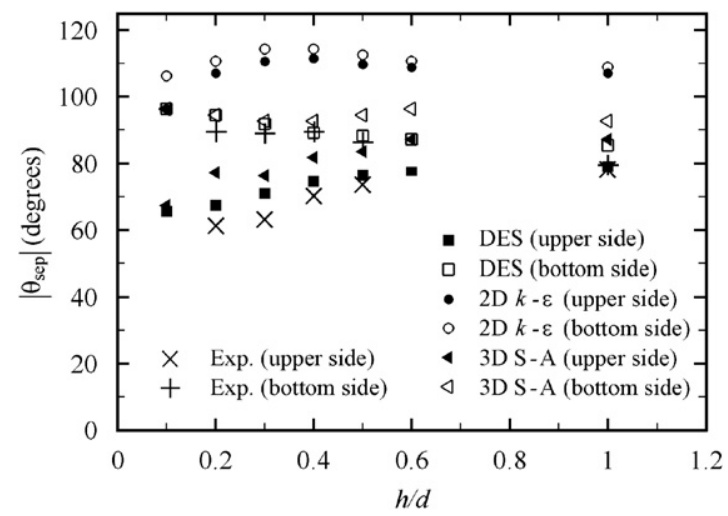

Fig. 12. Time-averaged separation angle versus gap ratio.

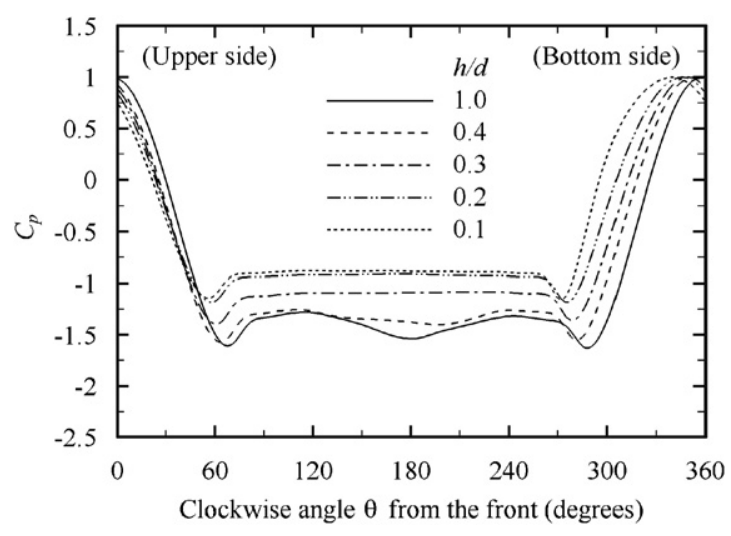

Fig. 13. Time-averaged pressure distributions (DES).

From the viewpoint of hydrodynamic stability theory of spatially developing flows, the key to the formation of von Kármán-type vortices behind two-dimensional bluff bodies is the existence of absolutely unstable regions in the nearwake region, which allows local disturbances to propagate both upstream and downstream, resulting in a resonance between the travelling instability waves [see Huerre and Monkewitz (1990) and Oertel (1990) for further descriptions]. Of interest here is that, a linear stability analysis of simplified wake profiles in ground effect (Nishino and Roberts, 


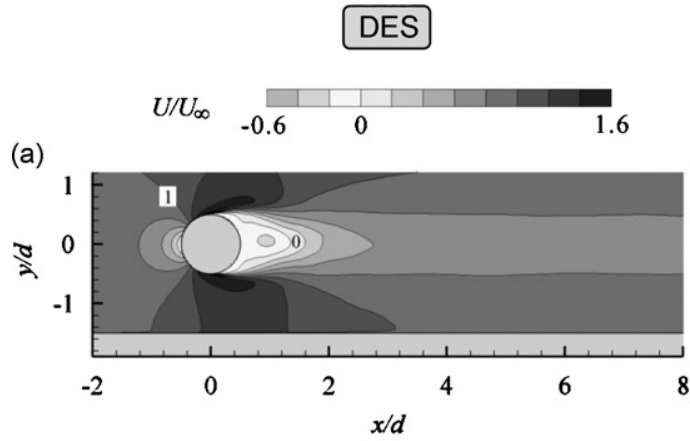

(b)

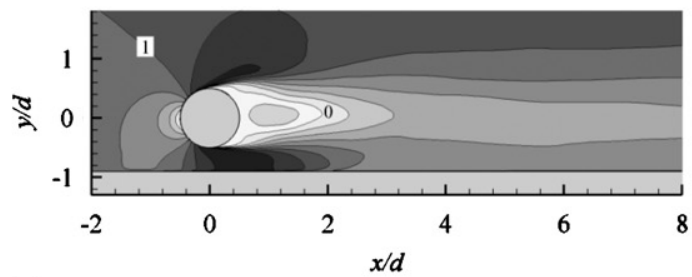

(c)

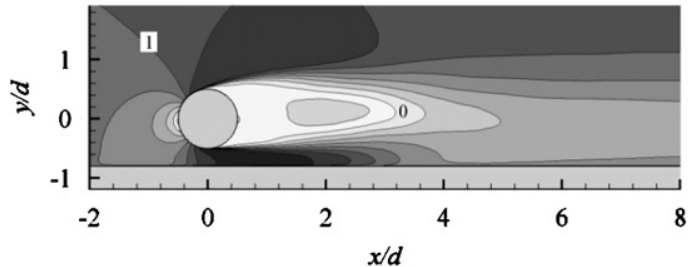

(d)

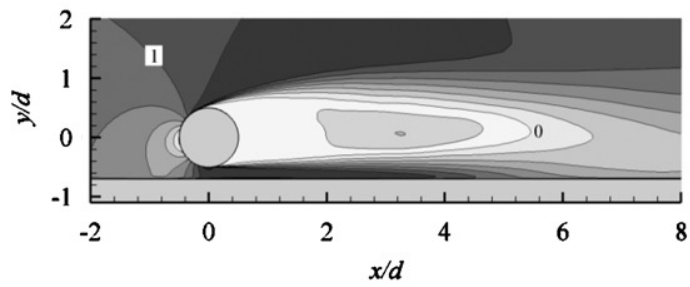

(e)

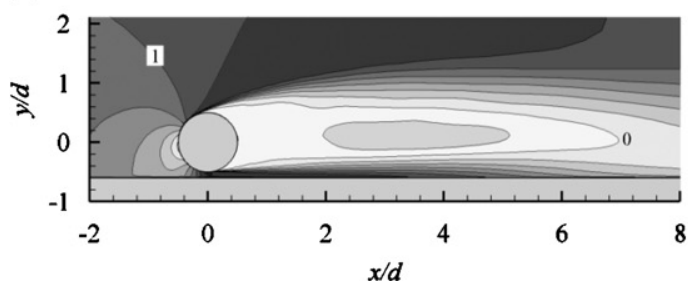

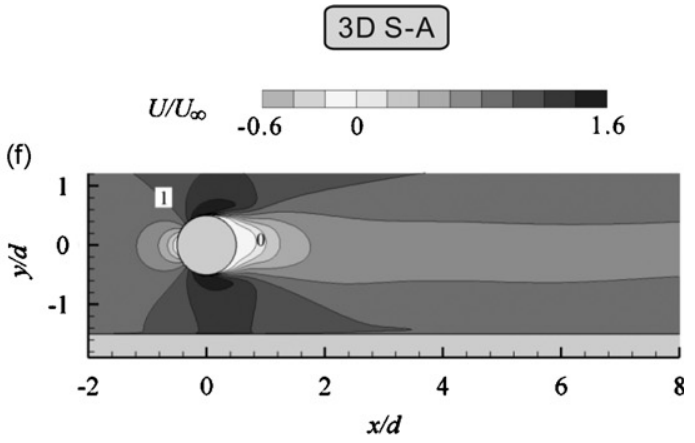

(g)

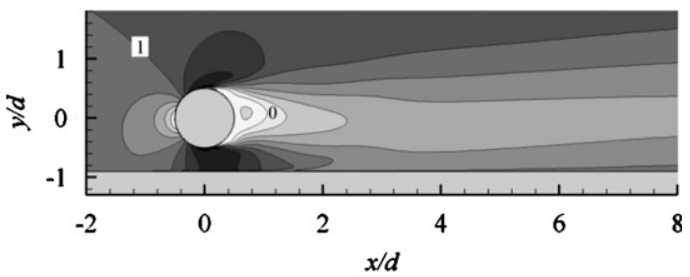

(h)

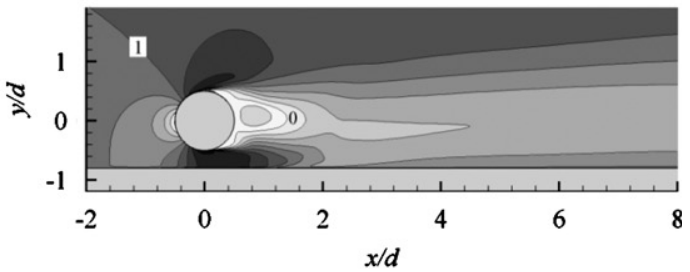

(i)

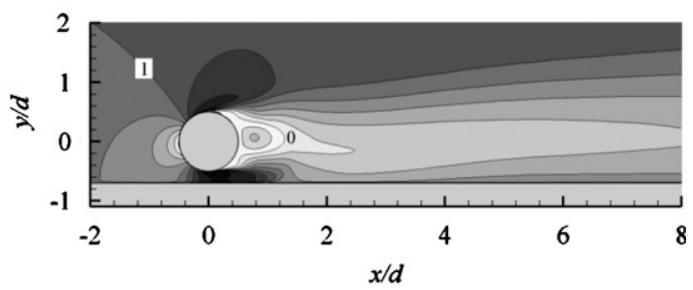

(j)

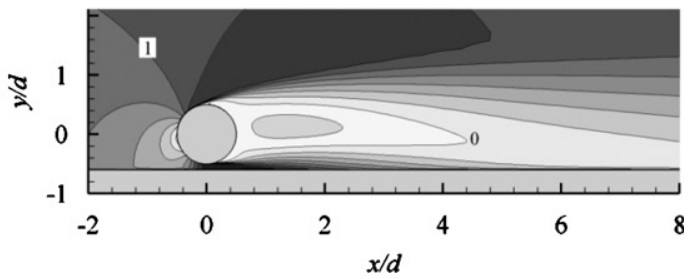

Fig. 14. Contours of nondimensional mean streamwise velocity: (a-e) DES, and (f-j) 3-D S-A. (a, f) $h / d=1.0 ;(\mathrm{b}, \mathrm{g}) h / d=0.4 ;(\mathrm{c}, \mathrm{h})$ $h / d=0.3 ;(\mathrm{d}, \mathrm{i}) h / d=0.2 ;(\mathrm{e}, \mathrm{j}) h / d=0.1$.

2007) indicated that the state of the wake tends to change from being absolutely unstable to being convectively unstable as the relative ground distance $H / B$, where $H$ is the distance from the wake centreline to the ground and $B$ is the width of the wake, is reduced, as well as when the reverse or backflow velocity of the wake is reduced. Comparing these trends with the results shown above in Fig. 17, it is speculated that the near-wake profiles predicted by the 3-D S-A URANS are more absolutely unstable than those predicted by the DES, and thus are more susceptible to the formation of the von Kármán-type vortices. 


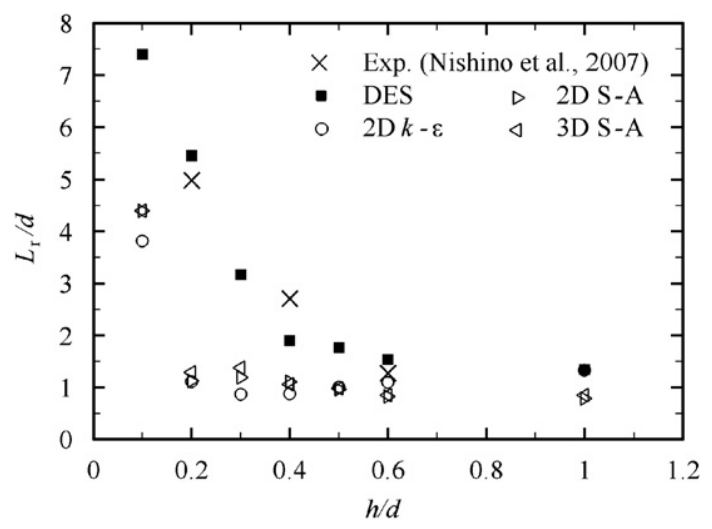

Fig. 15. Recirculation length versus gap ratio.

(a)

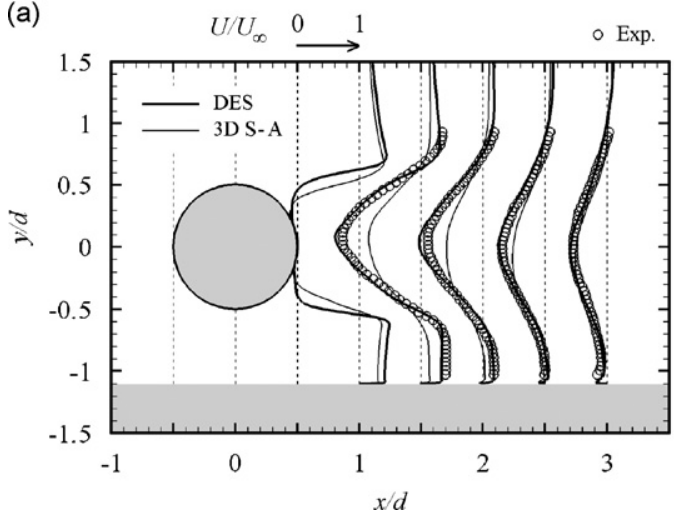

(b)

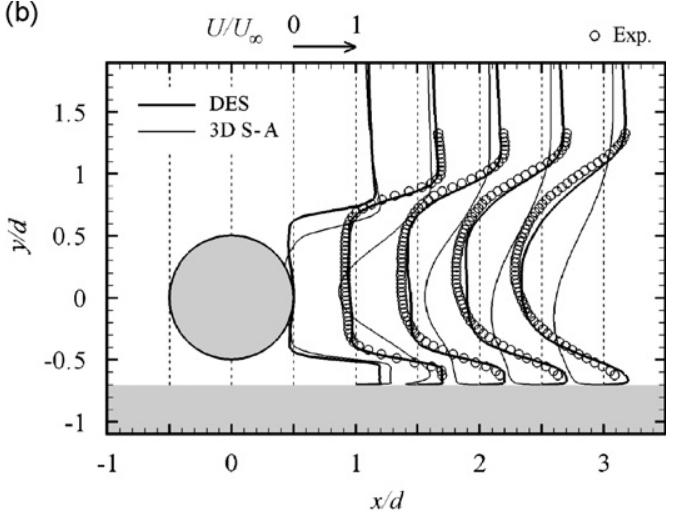

Fig. 16. Comparison of mean streamwise velocity profiles. (a) $h / d=0.6$; (b) $h / d=0.2$.

The above interpretation of the incorrect vortex shedding turns out to be more probable when one compares the present results with the 2-D URANS simulations of flow past a square cylinder placed near a fixed ground by Bosch and Rodi (1996), where the standard $k-\varepsilon$ model predicted an 'earlier' cessation of the vortex shedding in ground effect (i.e., the shedding was predicted to cease at a higher gap ratio than that observed in experiments), in contrast to the URANS simulations in the present study. Bosch and Rodi (1996) showed that the prediction of the cessation could be improved by employing an ad hoc modification of the $k-\varepsilon$ model proposed by Kato and Launder (1993) to prevent the excessive production of turbulence, and thus suggested that the 'earlier' cessation of the vortex shedding in ground effect predicted by the standard $k-\varepsilon$ model was due to the excessive damping caused by the excessive eddy viscosity in the wake. However, these results may also be interpreted, from the stability point of view, that the wider wake predicted by the standard $k-\varepsilon$ model was less absolutely unstable than the narrower wake predicted by the model with the Kato-Launder modification (note that the relative ground distance $H / B$ decreases as the wake width $B$ increases). That is to say, it is inferred that in the present study all URANS simulations predicted 'delayed' cessation of the vortex shedding behind a circular cylinder primarily because they predicted narrower wakes and therefore larger $H / B$ in the near-wake region, whereas in the study by Bosch and Rodi (1996) the standard $k-\varepsilon$ model predicted 'earlier' cessation of the vortex shedding behind a square cylinder primarily because the standard $k-\varepsilon$ model predicted wider wakes and therefore smaller $H / B$ in the near-wake region.

\section{Conclusions}

Unsteady RANS and detached-eddy simulations were performed for a subcritical flow $\left(\mathrm{Re}=4.0 \times 10^{4}\right)$ around a circular cylinder placed near and parallel to a moving ground. The DES, based on a modified S-A model, were 


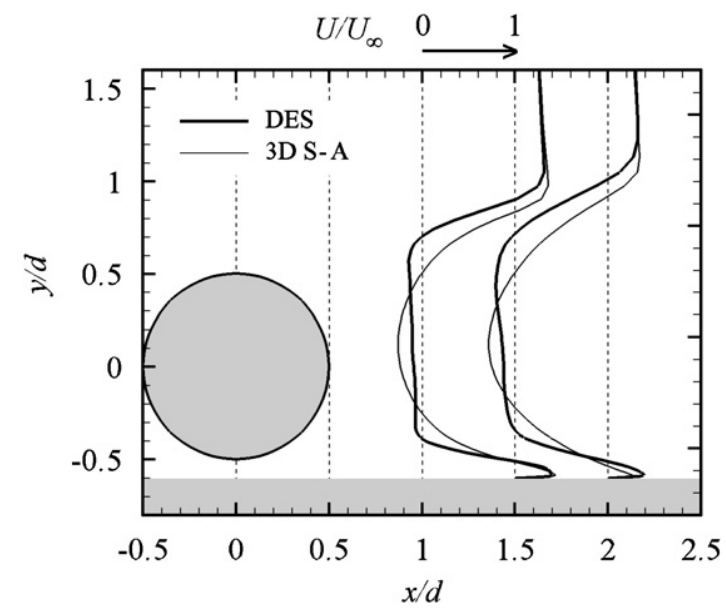

Fig. 17. Comparison of mean streamwise velocity profiles in the near-wake region $(h / d=0.1)$.

performed in a three-dimensional domain with periodicity imposed in the spanwise direction, whereas the URANS, employing the S-A model and also the LS $k-\varepsilon$ model, were conducted in both two- and three-dimensional domains. The simulations were performed at seven different gap ratios $(h / d)$ of $0.1,0.2,0.3,0.4,0.5,0.6$, and 1.0, and then compared with the experiments previously reported by the authors. The main findings are as follows.

First of all, the DES successfully captured the main features of the flow past the cylinder in ground effect, whereas the URANS did not. The DES predicted the cessation of the large-scale von Kármán-type vortex shedding behind the cylinder between two $h / d$ of 0.4 and 0.3 , which was consistent with the earlier experiments, whereas both two- and three-dimensional URANS also predicted it but at a much smaller $h / d$ of $0.2-0.1$. The wake structures of the cylinder predicted by the DES were in good agreement with the experiments in both large- and small-gap regimes, and also in the intermediate-gap regime, where the DES captured the intermittence of the large-scale vortex shedding in the near-wake region. The time-averaged drag coefficients, separation angles, and velocity profiles in the near-wake region predicted by the DES were also in good agreement with the experiments. These results may suggest the promise of DES to flows involving the problem of large-scale vortex shedding and its control or suppression often encountered in engineering applications.

Secondly, an interesting difference was also observed between the 2-D and 3-D S-A URANS solutions. That is, the former yielded fully periodic time variations in $C_{D}$ and $C_{L}$ at all $h / d$ of greater than 0.1 , whereas the latter showed some low-frequency modulations at $h / d \geqslant 0.4$, where moderately three-dimensional wake structures consisting of not only spanwise vortices but also streamwise 'rib' vortices were predicted. The vortical structures resolved in the 3-D S-A URANS, however, were still much coarser than those by the DES despite the same grid employed and hence almost the same computational costs required. As far as the time-averaged properties of the flow were concerned, the difference between the 2-D and 3-D URANS seemed less significant than those between the 3-D URANS and the DES.

Finally, the reason of the 'delayed' cessation of the vortex shedding in the URANS simulations was examined by comparing the mean wake profiles predicted at the smallest gap ratio of 0.1 , at which even the URANS captured the cessation of the vortex shedding. It was inferred that the near-wake profiles predicted by the URANS were more absolutely unstable than those predicted by the DES basically because the URANS predicted narrower wakes, which effectively increased the relative gap distance between the wake and the ground. Further investigations are needed, however, to fully understand the stability characteristics of wakes in ground effect, which may give more insight into the mechanisms of the cessation of von Kármán-type vortex shedding in URANS and DES as well as in real physics.

\section{Acknowledgment}

T. Nishino is supported by a research scholarship from the School of Engineering Sciences, University of Southampton, United Kingdom. 


\section{Appendix A. Influence of spatial and time resolution in DES}

Table 2 summarises the major results of preliminary computations that were performed to examine the influence of spatial and time resolution on the results (DES, $h / d=1.0$ ). Note that 'Baseline' in the table indicates the case with the spatial and time resolution that was eventually adopted in the main part of the study. Also note that the results of the URANS simulations on the same flow configuration $(h / d=1.0)$ are also listed at the bottom of the table for the purpose of comparison.

Cases A1, A2 ( = Baseline), and A3 compare the influence of spatial resolution in the $x$ and $y$ directions. In Case A1 the number of grid points in each $(x$ and $y$ ) direction was reduced by $40 \%$ from the baseline, whereas in Case A3 that was increased by $40 \%$ from the baseline. It can be seen from the results that the differences between Cases A2 and A3 are smaller than those between Cases A1 and A2, suggesting a certain degree of grid convergence in the $x$ and $y$ directions.

Cases B1, B2, B3 ( = Baseline), and B4 compare the influence of spatial resolution in the $z$ direction. Note that $\Delta z$ ( $2 d$ divided by the number of grid points in the $z$ direction) is the largest grid spacing (i.e., larger than $\Delta x$ and $\Delta y$ ) around the cylinder and thus directly affects the switching between the URANS and LES modes in that region in the DES in this study (cf. Section 2.4). Hence, compared with the thickness of the boundary layer around the cylinder (about $0.03 d$ at the maximum), the thickness of the URANS region around the cylinder $(0.65 \Delta z)$ is slightly larger in Case B3 ( = Baseline, 0.0325d), much larger in Cases B1 and B2 (0.13d and 0.065d, respectively), and smaller in Case B4 $(0.01625 d)$. It is therefore unsurprising that some discrepancies from the baseline are observed not only in Case B1, where the spanwise resolution is simply too coarse, but also in Case B4, where the switch between the URANS and LES modes undesirably takes place inside the boundary layer due to the fine spanwise resolution. Of interest, however, is that there is little difference in the results between Cases B2 and B3 (= Baseline). This shows that even in DES a certain degree of grid convergence could be achieved in an 'appropriate' resolution range, although this is not easy to be achieved for general industrial flows of complex geometry, as pointed out by Menter et al. (2003), who alternatively proposed a so-called scale-adaptive simulation (SAS) model to remedy the problem of the explicit grid dependency in DES.

Cases C1 ( = Baseline) and C2 compare the influence of time resolution on the results. Note that in Case C2 the time step size is reduced to one-fifth of that used in the baseline case. It is obvious from the results that the differences between the two cases are very small.

Table 2

Summary of computations with different spatial and time resolution $\left(\operatorname{Re}=4.0 \times 10^{4}, h / d=1.0\right)$

\begin{tabular}{|c|c|c|c|c|c|c|c|c|}
\hline Case & Grid $(x, y)$ & Grid $(z)$ & $\Delta t U_{\infty} / d$ & $C_{D}$ & St & $\theta_{\text {sep }}^{+}\left({ }^{\circ}\right)$ & $\theta_{\text {sep }}^{-}\left({ }^{\circ}\right)$ & $L_{r} / d$ \\
\hline DES (Baseline) & 35300 & 40 & 0.021 & 1.33 & 0.210 & 79.2 & 85.5 & 1.35 \\
\hline \multicolumn{9}{|c|}{ Different resolutions in $(x, y)$} \\
\hline A1 & 12708 & 40 & 0.021 & 1.03 & 0.242 & 86.1 & 87.9 & 1.44 \\
\hline A2 ( = Baseline) & 35300 & 40 & 0.021 & 1.33 & 0.210 & 79.2 & 85.5 & 1.35 \\
\hline A3 & 69188 & 40 & 0.021 & 1.41 & 0.200 & 81.4 & 87.0 & 1.21 \\
\hline \multicolumn{9}{|c|}{ Different resolutions in $z$} \\
\hline $\mathrm{B} 1$ & 35300 & 10 & 0.021 & 1.40 & 0.205 & 84.6 & 90.0 & 1.01 \\
\hline B2 & 35300 & 20 & 0.021 & 1.34 & 0.211 & 80.1 & 86.4 & 1.29 \\
\hline B3 ( = Baseline) & 35300 & 40 & 0.021 & 1.33 & 0.210 & 79.2 & 85.5 & 1.35 \\
\hline B4 & 35300 & 80 & 0.021 & 1.47 & 0.201 & 81.9 & 87.3 & 1.11 \\
\hline \multicolumn{9}{|c|}{ Different resolutions in time } \\
\hline $\mathrm{Cl}$ ( = Baseline $)$ & 35300 & 40 & 0.021 & 1.33 & 0.210 & 79.2 & 85.5 & 1.35 \\
\hline $\mathrm{C} 2$ & 35300 & 40 & 0.0042 & 1.32 & 0.212 & 80.1 & 84.6 & 1.36 \\
\hline \multicolumn{9}{|c|}{ Unsteady RANS results } \\
\hline $2-\mathrm{D} k-\varepsilon$ & 35300 & 1 & 0.021 & 0.74 & 0.292 & 107.1 & 108.9 & 1.34 \\
\hline 2-D S-A & 35300 & 1 & 0.021 & 1.50 & 0.237 & 89.1 & 93.6 & 0.79 \\
\hline $3-\mathrm{D} \mathrm{S}-\mathrm{A}$ & 35300 & 40 & 0.021 & 1.46 & 0.215 & 87.3 & 92.7 & 0.85 \\
\hline
\end{tabular}




\section{References}

Bearman, P.W., Zdravkovich, M.M., 1978. Flow around a circular cylinder near a plane boundary. Journal of Fluid Mechanics 89, 33-47.

Bosch, G., Rodi, W., 1996. Simulation of vortex shedding past a square cylinder near a wall. International Journal of Heat and Fluid Flow 17, 267-275.

Bosch, G., Rodi, W., 1998. Simulation of vortex shedding past a square cylinder with different turbulence models. International Journal of Numerical Methods in Fluids 28, 601-616.

Breuer, M., 1998. Numerical and modeling influences on large eddy simulations for the flow past a circular cylinder. International Journal of Heat and Fluid Flow 19, 512-521.

Breuer, M., 2000. A challenging test case for large eddy simulation: high Reynolds number circular cylinder flow. International Journal of Heat and Fluid Flow 21, 648-654.

Breuer, M., Jovičić, N., Mazaev, K., 2003. Comparison of DES, RANS and LES for the separated flow around a flat plate at high incidence. International Journal of Numerical Methods in Fluids 41, 357-388.

Ferziger, J.H., Peric, M., 2002. Computational Methods for Fluid Dynamics, third ed. Springer, Berlin (Chapter 9).

Fluent Inc., 2005. Fluent 6.2 User's Guide. Fluent Inc., Lebanon, NH, USA.

Huerre, P., Monkewitz, P.A., 1990. Local and global instabilities in spatially developing flows. Annual Review of Fluid Mechanics 22 , 473-537.

Hussain, A.K.M.F., Reynolds, W.C., 1970. The mechanics of an organized wave in turbulent shear flow. Journal of Fluid Mechanics 41, 241-258.

Kato, M., Launder, B.E., 1993. The modelling of turbulent flow around stationary and vibrating square cylinders. In: Proceedings of the 9th Symposium on Turbulent Shear Flows, Kyoto, pp. 10.4.1-10.4.6.

Launder, B.E., Sharma, B.I., 1974. Application of the energy-dissipation model of turbulence to the calculation of flow near a spinning disc. Letters in Heat and Mass Transfer 1, 131-137.

Lei, C., Cheng, L., Kavanagh, K., 1999. Re-examination of the effect of a plane boundary on force and vortex shedding of a circular cylinder. Journal of Wind Engineering Industrial Aerodynamics 80, 263-286.

Menter, F.R., Kuntz, M., Bender, R., 2003. A scale-adaptive simulation model for turbulent flow predictions. AIAA Paper 2003-0767, In: 41st AIAA Aerospace Sciences Meeting and Exhibit, Reno, NV, USA.

Nishino, T., Roberts, G.T., 2007. Absolute and convective instabilities of two-dimensional bluff body wakes in ground effect. European Journal of Mechanical B/Fluids, under review.

Nishino, T., Roberts, G.T., Zhang, X., 2007. Vortex shedding from a circular cylinder near a moving ground. Physics of Fluids 19, 025103.

Oertel Jr, H., 1990. Wakes behind blunt bodies. Annual Review of Fluid Mechanics 22, 539-564.

Patankar, S.V., 1980. Numerical heat transfer and fluid flow. Hemisphere, Washington, DC, USA.

Shur, M., Spalart, P., Strelets, M., Travin, A., 1996. Navier-Stokes simulation of shedding turbulent flow past a circular cylinder and a cylinder with backward splitter plate. In: Proceedings of the 3rd ECCOMAS Computational Fluid Dynamics Conference, 9-13 September, Paris, pp. 676-682.

Shur, M., Spalart, P.R., Strelets, M., Travin, A., 1999. Detached-eddy simulation of an airfoil at high angle of attack. In: Proceedingsof the 4th International Symposium on Engineering Turbulence Modelling and Measurements, Corsica. 24-26 May, Elsevier, Amsterdam, pp. 669-678.

Shur, M., Spalart, P.R., Squires, K.D., Strelets, M., Travin, A., 2005. Three dimensionality in Reynolds-averaged Navier-Stokes solutions around two-dimensional geometries. AIAA Journal 43, 1230-1242.

Spalart, P.R., 2000. Strategies for turbulence modelling and simulations. International Journal of Heat and Fluid Flow 21, $252-263$.

Spalart, P.R., 2001. Young-person's guide to detached-eddy simulation grids. Technical Report NASA/CR-2001-211032, Langley Research Center, National Aeronautics and Space Administration (NASA), Virginia, USA.

Spalart, P.R., Allmaras, S.R., 1992. A one-equation turbulence model for aerodynamic flows. AIAA Paper 92-0439.

Squires, K.D., 2004. Detached-eddy simulation: current status and perspectives. In: Friedrich, R., Geurts, B.J., Métais, O. (Eds.), Direct and Large-Eddy Simulation V. Kluwer Academic Publishers, Dordrecht, pp. 465-480.

Travin, A., Shur, M., Strelets, M., Spalart, P., 2000. Detached-eddy simulations past a circular cylinder. Flow, Turbulence and Combustion 63, 293-313.

Zdravkovich, M.M., 1985. Forces on a circular cylinder near a plane wall. Applied Ocean Research 7, $197-201$.

Zdravkovich, M.M., 1997. Flow Around Circular Cylinders: vol 1: Fundamentals. Oxford University Press, Oxford, UK. 